\title{
Activation of Arterial Matrix Metalloproteinases Leads to Vascular Calcification in Chronic Kidney Disease
}

\author{
Neal X. Chen ${ }^{a} \quad$ Kalisha D. O'Neilla Xianming Chen ${ }^{a} \quad K^{\prime}$ Kraiwiporn Kiattisunthorn ${ }^{c}$ \\ Vincent H. Gattone ${ }^{a}$ Sharon M. Moe ${ }^{a, b}$ \\ a Division of Nephrology, Indiana University School of Medicine, and boudebush Veterans Affairs Medical Center, \\ Indianapolis, Ind., USA; ${ }^{C}$ Faculty of Siriraj Medical School, Mahidol University, Bangkok, Thailand
}

\section{Key Words}

Matrix metalloproteinase $\cdot$ Gelatinase $\cdot$ Vascular

calcification $\cdot$ Chronic kidney disease

\begin{abstract}
Background: The objective of the current study was to determine if altered regulation of matrix metalloproteinases (MMPs) may predispose to extracellular matrix degradation, facilitating arterial calcification in chronic kidney disease (CKD) using a progressive model of CKD-MBD, the Cy/+ rat. Methods: Sera were collected from normal or CKD rats at various times and MMP-2 and MMP-9 levels determined by ELISA or zymography. Aorta tissue was harvested at sacrifice for RT-PCR and immunostaining. Calcification of aorta rings was assessed with MMP inhibitors. Results: There was an increase in MMP-2, MMP-9, TIMP-1, and RUNX-2 expression in the aorta with progressive CKD, and increased MMP-2 activity in the serum. Immunostaining revealed increased expression of MMP-2 and MMP-9 in areas of aorta calcification. There was also an upregulation of MMP-2 and MMP-9 in vascular smooth muscle cells (VSMC) from CKD rats. MMP inhibitors decreased calcification of aorta rings from normal and CKD rats. High phosphorus increased MMP-2 and MMP9 expressions in VSMC from normal rats but not from CKD rats. Conclusion: MMP-2 and MMP-9 expression and activity
\end{abstract}

are increased with progressive CKD, and blockade of MMP activity can inhibit arterial calcification. These data suggest degradation of the extracellular matrix is a critical step in the pathogenesis of arterial calcification in CKD.

Copyright $\odot 2011$ S. Karger AG, Basel

\section{Introduction}

Vascular calcification is common in patients with chronic kidney disease (CKD), diabetes, aging and inflammatory disease. The pathophysiology of vascular calcification is complex [1]. Vascular smooth muscle cells (VSMC) differentiate to osteochondrocytic cells capable of mineralization. These cells then synthesize matrix vesicles which interact with the extracellular matrix through a process that is poorly understood but appears to parallel bone modeling. During endochondral ossification, cartilage is transformed from an avascular tissue to a highly vascularized bone. During this complex process, metalloproteinases are critical through degradation of the extracellular matrix and by directing proliferation and communication between multiple cells [2]. Similar to bone, metalloproteinases have a critical role in arterial and cardiac remodeling $[3,4]$.

\section{KARGER}

Fax +41613061234 E-Mail karger@karger.ch www.karger.com
(ㄷ) 2011 S. Karger AG, Basel

0250-8095/11/0343-0211\$38.00/0

Accessible online at:

www.karger.com/ajn
Sharon M. Moe, MD

Department of Medicine, Indiana University School of Medicine

1001 West 10th Street, OPW 526

Indianapolis, IN 46202 (USA)

Tel. +1 317278 2868, E-Mail smoe@ @iupui.edu 
Matrix metalloproteinases (MMPs) are zinc- and calcium-dependent proteinases that degrade fibrillar and extacellular matrix proteins such as elastin and collagen through selective, but overlapping substrate specificities [5]. The gelatinases (gelatinase A is MMP-2, and gelatinase $B$ is MMP-9) have the most diverse substrates, degrading elastin, and collagen types IV, V, VII, and X [5]. The regulations of MMPs occur at several levels: synthesis of pro-MMPs, post-transcriptional conversion to active MMPs, and interaction with specific inhibitors [5]. MMP-2 is constitutively expressed in endothelial cells and VSMC whereas MMP-9 expression is more common in monocytes and other bone marrow-derived cells [4].

Serum levels of MMP-9 and MMP-2 are elevated in hemodialysis patients with a history of cardiovascular disease compared to those without disease and normal controls [6], and increased levels are associated with valvular calcification and atherosclerosis in the general population $[7,8]$. MMPs are also important in non-CKD animal models of arterial calcification [9]. We therefore hypothesized that altered regulation of MMPs in CKD leads to medial artery extracellular matrix degradation, accelerating arterial calcification in CKD. To test this hypothesis, we used a slowly progressive model of CKD$\mathrm{MBD}$, the $\mathrm{Cy} /+$ rat that develops spontaneous hyperphosphatemia, hyperparathyroidism and vascular calcification [10].

\section{Methods}

\section{Animal Models and Tissue Harvest}

In the present study, we utilized the Han:SPRD Cy/ $+_{\text {IU }}$ rat with autosomal dominant polycystic kidney disease [11]. This is an autosomal dominant condition, such that at birth, $1 / 4$ of the animals are normal $(+/+), 1 / 2$ are heterozygotes $(\mathrm{Cy} /+)$, and $1 / 4$ are affected homozygotes (Cy/Cy). Homozygous Cy/Cy rats develop massively enlarged kidneys and severe azotemia, and normally die by 4 weeks of age. The male $\mathrm{Cy} /+$ rat develops a persistent azotemia starting at about 10 weeks of age, which progresses to terminal uremia by about 40 weeks, with spontaneous and slow development of all three manifestations of CKD-MBD: biochemical abnormalities, extraskeletal calcification, and abnormal bone [11]. For the present study, weaned rats were housed in open-top, shoebox cages, and had free access to tap water and standard chow until they were 19 weeks old when they were switched from a standard pellet rat chow to a powdered diet of $18 \%$ casein-based protein, $0.7 \%$ phosphorus, $0.7 \%$ calcium, $5 \%$ fat (Harlan Teklan TD.04539). The animals (Cy/+ rat and normal littermate +/+, hereafter called CKD and normal rat) had blood drawn at 20,29 and 34 weeks by tail bleed. At sacrifice ( 38 weeks), rats were anesthetized with sodium pentobarbital $(50 \mathrm{mg} / \mathrm{kg}$ i.p.) and blood was collected by cardiac puncture. Thoracic aortas were collected and stored at $-80^{\circ} \mathrm{C}$. All procedures were reviewed and approved by the Indiana University School of Medicine Institutional Animal Care and Use Committee.

Serum MMP-2 and TIMP-1 Measurements

Serum levels of MMP-2 and TIMP-1 were determined by ELISA (R\&D Systems, Minneapolis, Minn., USA).

MMP Gelatin Zymography

MMP activity was assessed using the methods of El Bedoui et al. [12]. Serum from normal or CKD rats and conditioned media from aorta ring cultures that had been incubated with inhibitors of MMP activity were analyzed by $10 \%$ SDS-PAGE containing $0.1 \%$ gelatin. Gels were stained with Coomassie Brilliant Blue (0.2\%) and destained in $40 \%$ methanol and $10 \%$ acetic acid. MMP2 and MMP-9 standards (Chemicon, Temecula, Calif., USA) were run in parallel to confirm accurate molecular weight. The bands were analyzed by densitometry using a GelDoc Imaging System (Bio-Rad, Hercules, Calif., USA).

\section{Real-Time (Quantitative) RT-PCR Analysis}

Total RNA was isolated from frozen aorta using TRIzol Reagent according to the manufacturer's directions (Invitrogen, Carlsbad, Calif., USA). The MMP-2, MMP-9, TIMP-1, and RUNX-2 expression in rat aorta tissues or VSMC was determined by real-time RT-PCR. Reverse transcription reactions were performed with $2 \mu \mathrm{g}$ of total RNA using TaqMan reverse transcription reagent (Applied Biosystems, Foster City, Calif., USA). Target-specific PCR primers were obtained from Applied Biosystems. Real-time PCR amplifications were performed using TaqMan Gene Expression Assays (TaqMan MGP probes, FAM dye-labeled) using Applied Biosystems 7500 Real-Time PCR systems (Applied Biosystems). The cycle number at which the amplification plot crosses the threshold was calculated $\left(\mathrm{C}_{\mathrm{T}}\right)$, and the $\Delta \Delta \mathrm{C}_{\mathrm{T}}$ method are used to analyze the relative changes in gene expression using $\beta$-actin as a housekeeping gene.

Histology and Immunohistochemistry of Aortas from Normal and CKD Rats

Aorta tissue was harvested at 38 weeks from normal or CKD rats and processed in paraffin sections. Vessels were stained for the presence of calcification by MacNeal's staining as previously described [13]. Briefly, sections are placed in $0.2 \%$ silver nitrate for $10 \mathrm{~min}$ in the dark, rinsed, placed in sodium carbonate-formaldehyde, followed by Farmer's diminisher and counterstaining with tetrachrome solution. The expression of MMP-2 and MMP9 was examined by immunohistochemistry [13]. The sections were incubated with anti-MMP-2 or -MMP-9 antibodies (1:100; Santa Cruz Biotechnology, Santa Cruz, Calif., USA) and followed by ABC staining systems (Santa Cruz Biotechnology) with 3',3diaminobenzaidine tetrahydrochloride (DAB) for color development. The sections were counterstained with hematoxylin. Negative controls were obtained by substituting the primary antibody with PBS. Images were evaluated and recorded on a digital camera (Nikon Coolpix 950) using a Nikon Eclipse E400 microscope through a $20 \times$ or $40 \times$ objective (1.4 numerical aperture).

Organ Culture

Segments of rat thoracic aorta measuring 3-5 $\mathrm{cm}$ were harvested from 38-week-old normal or CKD rats and gently cleared of surrounding tissues. Aortic segments were incubated in serum-
Chen/O'Neill/Chen/Kiattisunthorn/ Gattone/Moe 


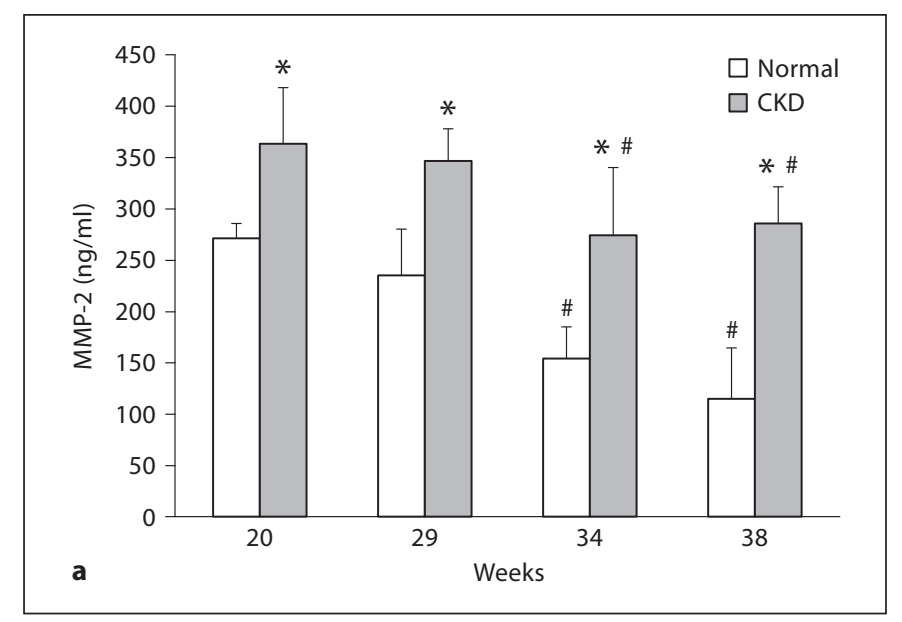

Fig. 1. MMP-2 serum levels and activity are increased in CKD rats CKD rats at 20,29, 34 and 38 weeks, and serum MMP-2 levels were determined by ELISA. The results demonstrate serum MMP-2 levels are significantly increased in CKD rats compared to normal rats at all time points (a), with no increase over time. Serum MMP-2 compared to normal rats: blood was collected from normal and

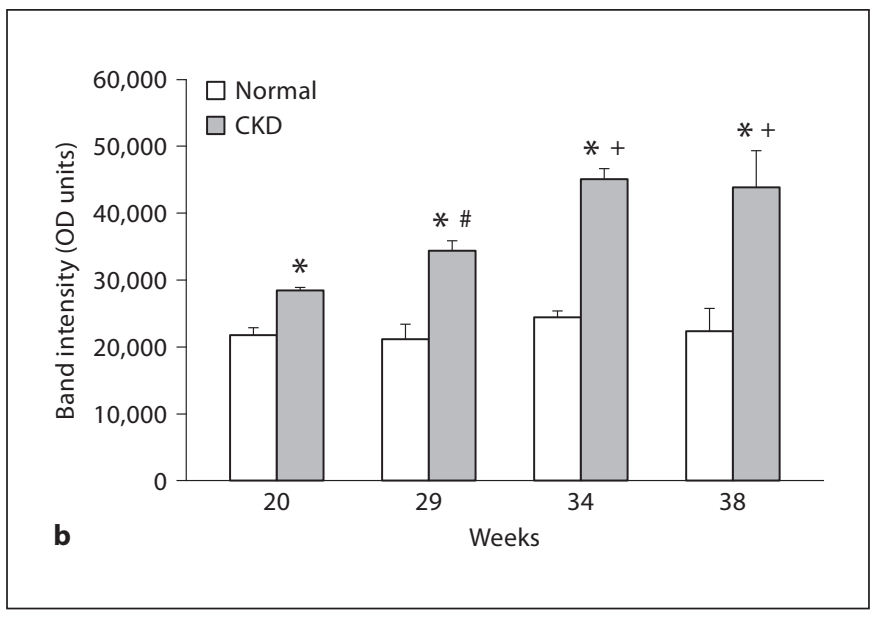

activity was further assessed by zymography, and the results demonstrated active MMP-2 was increased in CKD compared to normal rats at all time points and increased over time with the progression of CKD (b). Data are shown as mean $\pm \mathrm{SD}$ ( $\mathrm{n}=6$ each group). ${ }^{*} \mathrm{p}<0.05$, different from normal, same age; ${ }^{\#,+} \mathrm{p}<0.05$, different from age, normal or CKD, respectively, by two-way ANOVA.

\section{Results}

Louis, Mo., USA) containing penicillin/streptomycin at $37^{\circ} \mathrm{C}$ in a humidified $5 \% \mathrm{CO}_{2} / 95 \%$ incubator. Calcification was induced with the addition of 7.5 units/ml of calf intestinal alkaline phosphatase and $3.8 \mathrm{~mm}$ sodium phosphate (calcification medium) according to the methods of O'Neill's group [14]. To determine the role of MMPs in aorta calcification ex vivo, various concentrations of the MMP inhibitor GM6001 or its negative control (EMD, La Jolla, Calif., USA) and doxycycline (Sigma) were pre-equilibrated in medium and then added to the culture medium. Culture medium was changed every 2-3 days. After 7 days, conditioned media were collected for zymography. Aortic specimens were decalcified in $0.6 \mathrm{~N} \mathrm{HCl}$ for $72 \mathrm{~h}$. The calcium content of $\mathrm{HCl}$ supernatants was determined colorimetrically and normalized by tissue weight as previously described [15]. Viability of aorta organ cultures was determined by lactic dehydrogenase secretion into the medium using CytoTox-One Homogeneous Membrane Integrity Assay (Promega Inc., Madison, Wisc., USA).

\section{Cell Culture}

Rat VSMC (RVSMC) were isolated from the descending thoracic aorta in the normal or $\mathrm{Cy} /+(\mathrm{CKD})$ rats by the explant method as previously described [15]. The RVSMC were grown in DMEM (Sigma), with $10 \%$ FBS until confluent at which time they were replated for specific experiments. Cells were treated with normal or high phosphorus for $24 \mathrm{~h}$ and total RNA isolated for RT-PCR to determine MMP-2 and MMP-9 expression.

\section{Statistical Analyses}

Analyses were performed by paired t test or two-way ANOVA to examine the effect of disease (CKD vs. normal) and age (time). All results are presented as mean $\pm \mathrm{SD}$ and all analyses were done on StatView (SAS, Cary, N.C., USA).

MMP and Vascular Calcification

\section{Serum MMP-2 Levels and Activity Are Significantly Increased in CKD Animals Compared to Normal Animals}

Blood was collected at 20,29, 34 and 38 weeks from normal and CKD rats and serum MMP-2 levels measured by ELISA. The results demonstrated that the serum MMP2 level is significantly increased in CKD rats compared to normal rats at each time point examined (fig. 1a; $p<0.05$ ). MMP-2 levels were lower at later time points (34 and 38 weeks) compared to early time points (20 and 29 weeks) in both normal and CKD rats. Since ELISAs only measure the total MMP, which includes both the pro and the activated form of MMP, and there is no MMP-9 assay that works in rats, we performed zymography to determine the gelatinase activity. The results demonstrated increased MMP-2 activity in CKD compared to normal rats at each time point (fig. $1 b ; p<0.05$ ), and a progressive rise in MMP-2 activity by zymography as the animals age (fig. 1b; $\mathrm{p}<0.05)$. Thus, there is a progressive increase in active MMP-2 levels and activity in the serum of CKD rats compared to normal animals. However, no detectable MMP-9 activation was observed by zymography in rat serum from normal or CKD animals. We also analyzed serum TIMP1 levels and found levels were elevated in CKD animals compared to normal animals (fig. $2 ; \mathrm{p}<0.05$ ), but there was no significant change over time in either group. 
Fig. 2. TIMP-1 serum levels are increased in $\mathrm{CKD}$ rats compared to normal rats: blood was collected from normal and CKD rats at 20, 29, 34 and 38 weeks, and serum TIMP-1 levels were determined by ELISA. The results demonstrated serum TIMP-1 levels are increased in CKD rats compared to normal rats at all time points, but there was no change with increasing age/progression of CKD. Data are shown as mean $\pm \mathrm{SD}$ ( $\mathrm{n}=6$ each group). ${ }^{*} \mathrm{p}<$ 0.05 , different from normal.

Fig. 3. mRNA expression of MMP-2, MMP-9, TIMP-1, and RUNX-2 is increased in the thoracic aorta from 38 -weekold CKD rats: thoracic aortas were harvested at 38 weeks of age from CKD and normal animals and the expression of MMP-2 and MMP-9 was determined by RT-PCR. The results demonstrated increased expression of MMP-2 (a), MMP-9 (b), TIMP-1 (c) and RUNX-2 (d) in aortas from CKD rats compared to that in normal rats. Data are shown as mean \pm SD ( $\mathrm{n}=6$ each group). ${ }^{*} \mathrm{p}<0.05$, different from normal.
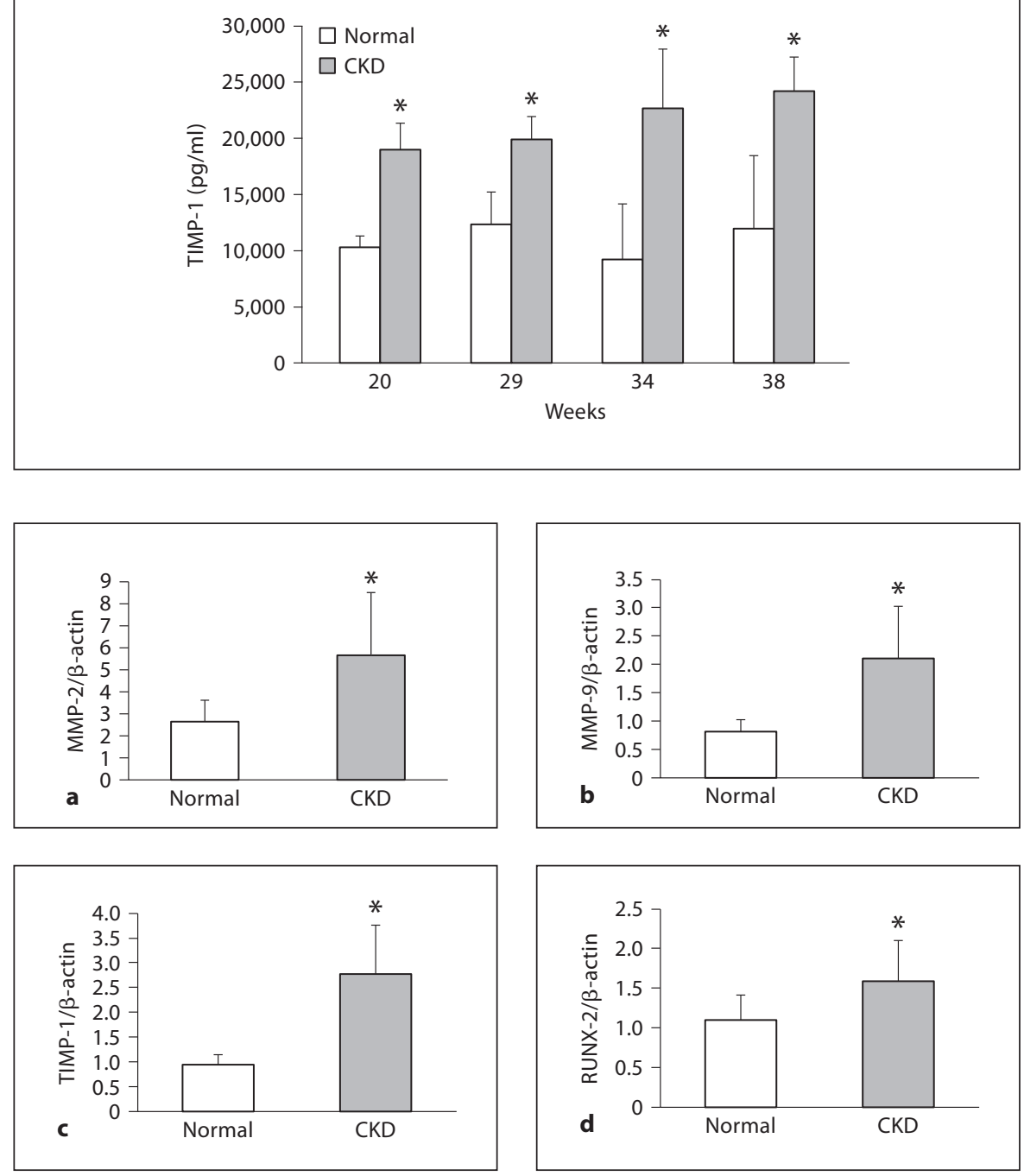

mRNA Expression of MMP-2 and MMP-9 Is

Increased in the Aorta of CKD Animals

Thoracic aortas from CKD and normal animals were harvested at 38 weeks of age, and the expression of MMP2 and MMP- 9 was determined by real-time RT-PCR. The results demonstrated that there is increased expression of MMP-2 (fig. 3a), MMP-9 (fig. 3b), TIMP-1 (fig. 3c), and RUNX-2 (fig. 3d) in aorta from CKD rats compared to that in normal rats.

Immunostaining Revealed Upregulation of MMP-2 and MMP-9 in Areas of Medial Calcification of CKD Rats

Aorta tissue was harvested at sacrifice (week 38) from normal or CKD rats and processed for histology (MacNeal's staining for calcification and immunohistochem- istry for MMP-2 and MMP-9 expression. Five sections from each group were analyzed, and 3 of the CKD animals had extensive calcification and the other 2 had minor 'dots' of calcification. None of the normal animals had evidence of calcification. Representative sections (fig. 4) of two of the vessels with more extensive medial calcification from CKD rats are shown in figure $4 \mathrm{~b}$ and $c$, and one of the aortas from a normal rat is shown in figure 4a. The expression of MMP-9 is upregulated in aorta from CKD rats (fig. 4 f, g) compared to minimal expression in normal littermates (fig. 4e). MMP-9 expression is seen in areas near or around calcification (fig. 4d, enlarged from box in fig. 4g). Similarly, MMP-2 expression also increased in aorta from CKD rats (fig. $4 \mathrm{j}, \mathrm{k}$ ) compared to that in normal rats (fig. 4i), although there 

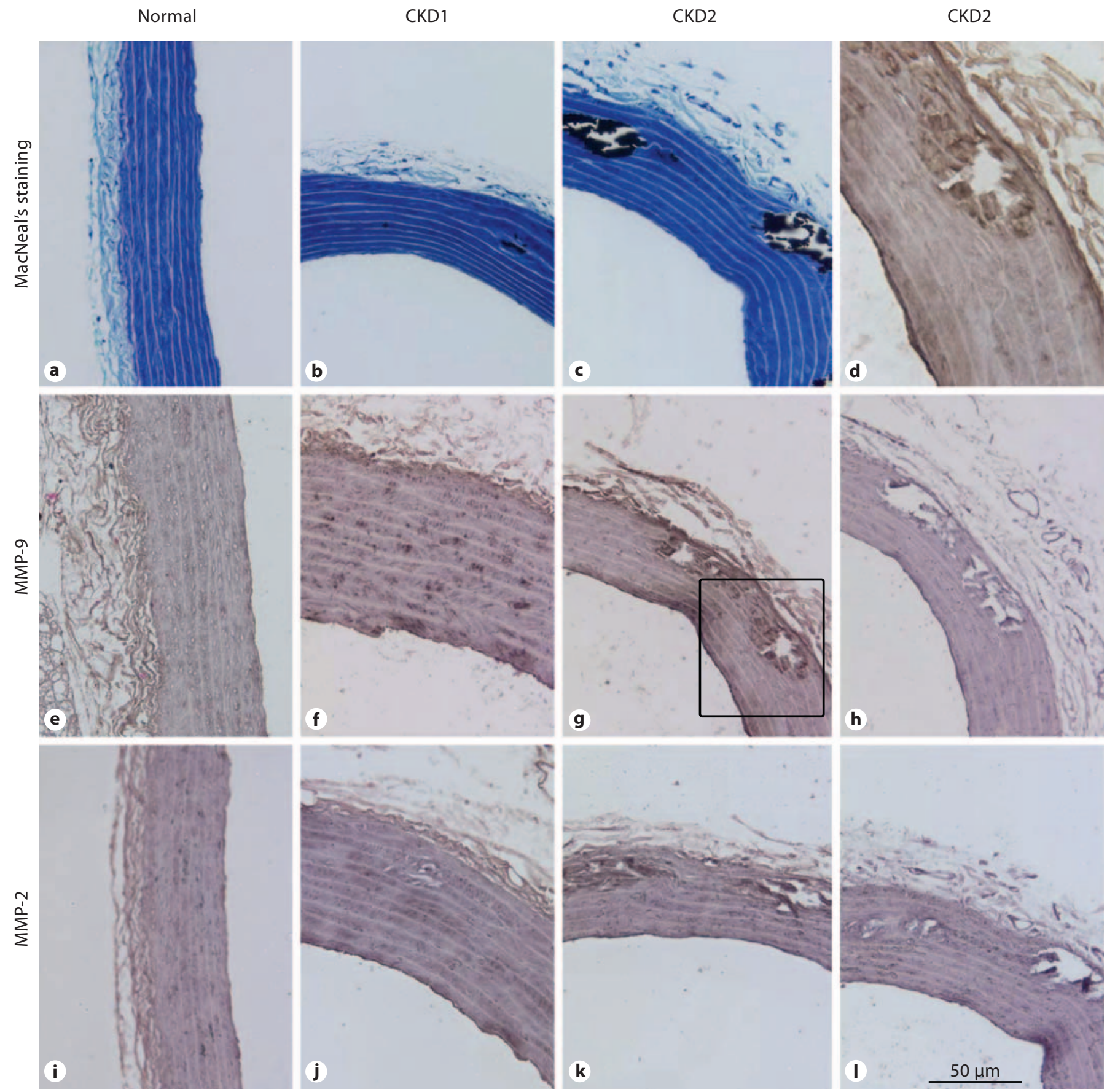

Fig. 4. Histology and immunohistochemistry of aorta in normal and CKD rats: aorta tissue was harvested at sacrifice (38 weeks) from normal or CKD rats and processed for histology (MacNeal's staining for calcification) and immunohistochemistry for MMP2 and MMP-9 expression. Two of the vessels with more extensive medial calcification from CKD rats are shown $(\mathbf{b}, \mathbf{c})$ and one of the aortas from a normal rat is shown (a). The expression of MMP9 is upregulated in aorta from CKD rats $(\mathbf{f}, \mathbf{g})$ compared to mini-

mal expression in normal littermates (e). MMP-9 expression is seen in areas near or around calcification (d, enlarged from box in g). Similarly, MMP-2 expression in also increased in aorta from CKD rats $(\mathbf{j}, \mathbf{k})$ compared to that in normal rats (i), although there is more basal expression of MMP-2 in both CKD and normal animals. The negative controls for MMP-9 and MMP-2 are shown in $\mathbf{h}$ and $\mathbf{I}$. 
is more basal expression of MMP-2 in both CKD and normal animals. The negative control for MMP-9 and MMP2 are shown in figure $4 \mathrm{~h}$ and 1.

\section{Inhibition of MMP Activity Decreased Aorta Calcification ex vivo}

To confirm the importance of MMP-2 and MMP-9 in the pathogenesis of vascular calcification, we used the aorta ring culture model which leads to calcification in the presence of sodium phosphate and alkaline phosphatase, the latter to inhibit naturally occurring pyrophosphate. The aorta rings were incubated with calcification media in the presence of different concentrations of various MMP inhibitors, GM6001 as well as its negative control, and doxycycline and MMP activity determined by zymography from the media. GM6001 inhibited the activation of MMP-2, doxycycline completely inhibited both the pro and active forms of MMP-2 (fig. 5a). However, the negative control for GM6001 had no effect on MMP activity. There was no detectable pro or active MMP-9 by zymography in the media from aorta ring cultures. The aorta rings were also analyzed for calcification by biochemical analysis. The results demonstrated that inhibition of MMP activity with GM6001 or doxycycline significantly decreased calcification in aorta rings, whereas GM6001-negative control had no effect (fig. 5b). These results suggest that MMP-2 activation is involved in the pathogenesis of aorta calcification. For the above experiments, we used aortas harvested from normal animals. To determine if MMP inhibitors also decrease calcification in CKD rats, we similarly incubated aortic ring cultures from CKD rats with GM6001 and doxycycline, demonstrating a reduction in calcification from control of 42 and $50 \%$, respectively.

\section{Elevated Serum Phosphorus May Contribute to}

Upregulation of MMP-2 and MMP-9 in CKD

To determine the potential mechanism of altered expression of MMPs in VSMC from the medial layer of the aortas, we isolated VSMC from normal and CKD rats and incubated the cells with normal or high phosphorus for $24 \mathrm{~h}$ and MMP-2 and MMP-9 expression determined by RT-PCR. The results demonstrated that both basal MMP2 (fig. 6a) and MMP-9 (fig. 6b) expression was increased in VSMC from CKD rats compared to that from normal rats $(\mathrm{p}<0.05)$. High phosphorus increased MMP-2 and MMP-9 expression in VSMC from normal rats, but had no effect on MMP-2 and MMP-9 expression in VSMC from CKD rats (fig. 6a, b). In contrast, parathyroid hormone did not increase basal expression of either MMP-2

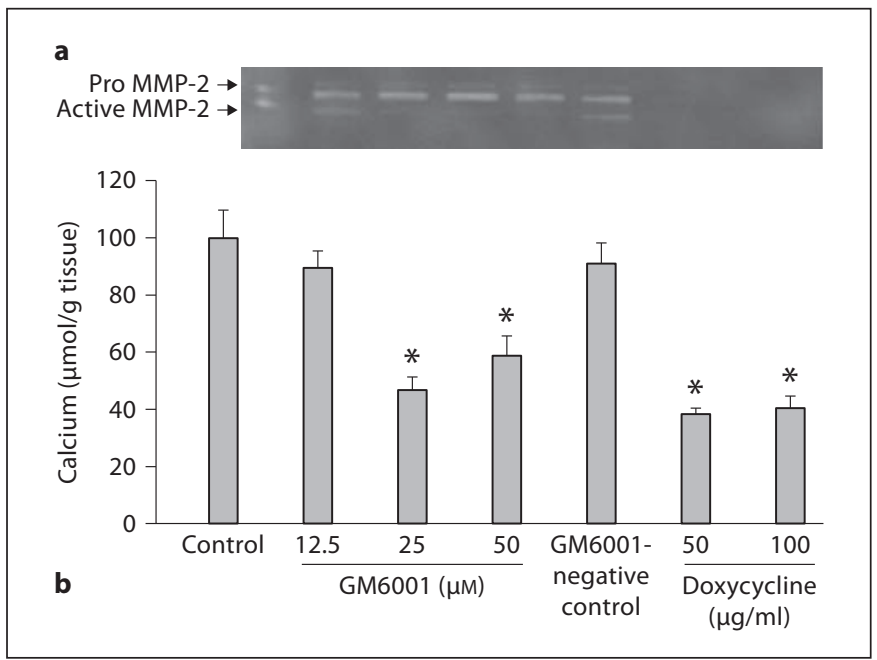

Fig. 5. Inhibition of MMP activity reduced calcification of rat aorta ring cultures: rat thoracic aorta rings were incubated with calcification media in the presence of MMP inhibitors for 7 days. Conditioned media was assessed for MMP activity by zymography, and aorta calcification determined biochemically. The results demonstrated that both MMP inhibitors GM6001 and doxycycline decreased MMP-2 activity (a) and reduced aorta ring calcification (b), whereas GM6011-negative control had no effect. Data are shown as mean $\pm \mathrm{SD}(\mathrm{n}=3$ from a total of 3 rats $) .{ }^{*} \mathrm{p}<$ 0.05 vs. control.

of MMP-9 in VSMC from normal or CKD rats (data not shown). However, zymography failed to show any difference with phosphorus or parathyroid hormone and thus we could not confirm that the increased expression led to increased activation.

\section{Discussion}

The current study demonstrated a role of MMP-2, and to a lesser extent MMP-9, in arterial calcification. Using the $\mathrm{Cy} /+$ rat, a spontaneous and slowly progressive model of CKD-MBD, we demonstrated increased MMP-2, MMP-9, and TIMP-1 expression by RT-PCR in the aorta with progressive CKD, and increased MMP-2 activity in the serum. This increase in enzyme expression was observed at the same time as increased expression of RUNX-2, an osteoblast transcription factor thought to be important in the calcification initiating step of osteochondrocytic differentiation of VSMC [1]. The expression was confirmed by immunohistochemistry to be upregulated in areas of calcification in CKD rats, although some basal expression of MMP-2 was also observed as previ- 


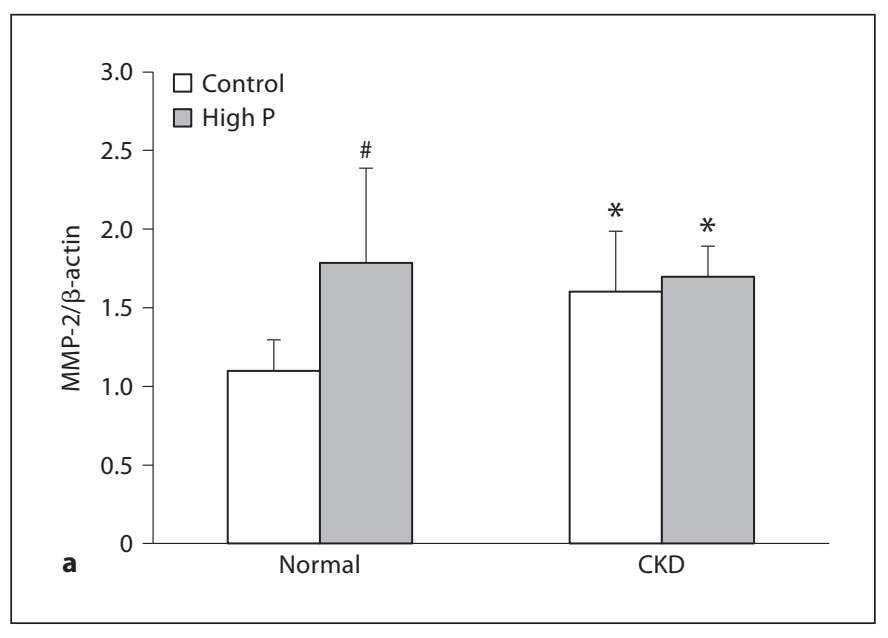

Fig. 6. Effect of phosphorus on MMP-2 and MMP-9 expression in VSMC from normal or CKD rats: VSMC isolated from normal or CKD rats were treated with control $(0.9 \mathrm{mM})$ or high phosphorus (P; $3 \mathrm{~mm}$ ) for $24 \mathrm{~h}$, and total RNA was isolated for RT-PCR for MMP-2 and MMP-9 expression. The results demonstrated that both MMP-2 (a) and MMP-9 (b) expression was upregulated in

ously reported in both normal and CKD animals [4]. We further demonstrated upregulation of both MMP-2 and MMP-9 in cultured cells from CKD rats compared to normal rats. To demonstrate a direct relationship of MMPs and arterial calcification, we used an ex vivo aorta ring culture model. Both a general inhibitor of MMPs, doxycycline, and a specific inhibitor of MMPs, GM6001 (which inhibits MMP-1, -2, -3, -8 and -9), decreased arterial calcification in normal and CKD aortas. Taken together, the data suggest that degradation of the extracellular matrix is a critical step in the pathogenesis of arterial calcification in CKD.

The expression of MMPs is critical for the normal bone remodeling that occurs throughout life [2]. It is therefore not surprising that MMPs are also important in the pathogenesis of extraosseous calcification. There is increased MMP-2 and MMP-9 in calcified aortic valves compared to non-calcified valves $[7,8]$. In human coronary arteries, MMP-9 activation was associated with increased calcification of atherosclerotic plaques [16]. In an animal model of medial calcification induced by direct administration of calcium chloride to the artery, calcium itself led to disruption and calcification of the elastin fibrils in a dose- and time-dependent process. However, neither elastin fragmentation nor calcium deposition occurred with this procedure in MMP-2 or MMP- 9 knockout animals [17]. In rats with arterial calcification in-

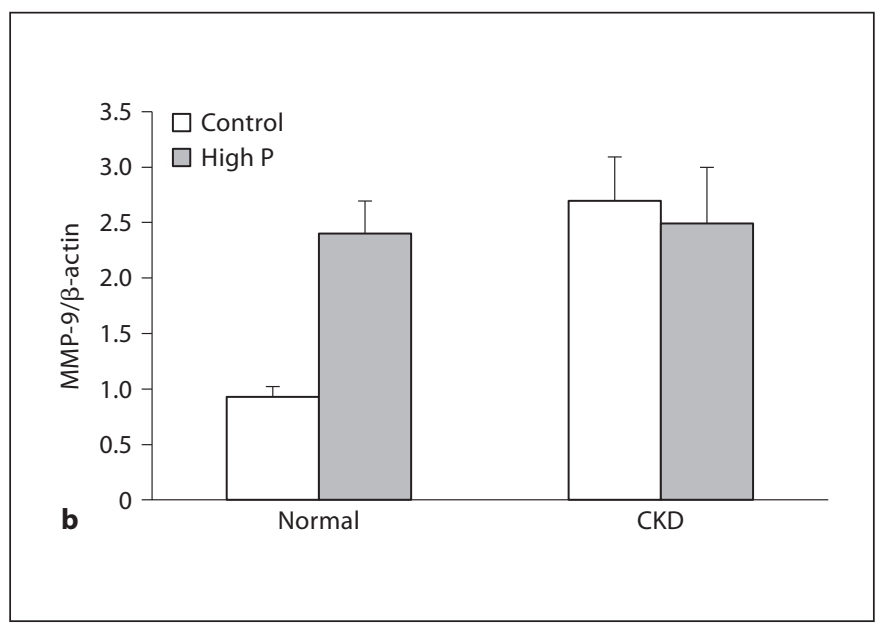

VSMC from CKD rats compared to that from normal rats. High phosphorus increased MMP-2 and MMP-9 expressions in VSMC from normal rats but had no effect on MMP expression in VSMC from CKD rats $(\mathbf{a}, \mathbf{b})$. Data are shown as mean $\pm \mathrm{SD}(\mathrm{n}=3)$. ${ }^{*} \mathrm{p}<0.05$, CKD compared to normal; ${ }^{*} \mathrm{p}<0.05$, high phosphorus compared to control phosphorus in the same group.

duced by high-dose vitamin $\mathrm{D}_{3}$ or periadventitial calcium chloride administration, or in ex vivo aortic ring cultures from normal rats, doxycycline and GM6001 could inhibit calcification [9].

Our data extend the above findings to the setting of CKD. We hypothesized that the degradation of extracellular matrix is a necessary, and early, step for subsequent arterial calcification. Differences in serum levels of activated MMP-2 were apparent in the Cy/+ (CKD) rat compared to normal rats as early as 20 weeks, an age at which there is a $50 \%$ reduction in kidney function and increased progressively thereafter. Furthermore, there was also increased tissue expression of MMP-2 and MMP-9 in CKD rats compared to controls. There were also increased levels of TIMP-1 in CKD animals compared to normal animals, perhaps to compensate for the increased MMPs expression. However, there was no progressive increase in TIMP-1 as there was for MMP-2 activation in the CKD animals, and thus this regulatory mechanism could not fully compensate with deteriorating CKD.

Our findings parallel studies in humans with CKD. In a cross-sectional study of arterial samples from patients undergoing a kidney transplant, there was increased expression of MMP-2 with increasing calcification [18]. In another study, the authors also found that both MMP-2 and MMP-9 had increased activity by zymography in CKD patients with diabetes compared to those without 
diabetes and the magnitude of expression correlated with stiffness and the phosphate concentration [19]. Finally, in dialysis patients, elevated MMP-2 levels were significantly correlated with increased carotid intimal-medial thickness, and serum levels of MMP-2 and MMP-9 are higher in hemodialysis patients with a history of cardiovascular disease compared to those without disease and normal controls [6].

Our animal and aortic ring cultures are consistent with these cross-sectional human studies and further demonstrate a progressive increase in MMP-2 expression with progressive CKD. We hypothesized that degradation occurred prior to the onset of calcification, and the current study supports this sequence of events, as the upregulation of MMPs was apparent at 20 weeks (50\% normal GFR) in this model, whereas we only see calcification after 30 weeks [11]. Thus, the degradation of the extracellular matrix is likely an important initial factor in 'preparing' the arteries for calcification. In addition to the degradation of extracellular matrix proteins, MMP-2 and MMP-9 can cleave different cytokines, chemokines, growth factors, and endothelin-1 (reviewed by Back et al. [4]), further contributing to the cellular differentiation of VSMC known to occur early in the pathogenesis of arterial calcification. While ECM degradation is clearly a critical step in medial calcification, other factors, such as the presence of inhibitors, e.g. matrix gla protein and fetuin-A, also play an important regulatory role [1]. Based on our study and others in the literature, MMP inhibitors such as TIMP-1 can now also be added to the list of regulators of arterial calcification, although our data indicate that they are inadequate to stop MMP matrix degradation.

Our data suggest that the altered regulation of MMPs in CKD is due to transcriptional regulation of both MMP2 and MMP-9, and post-transcriptional activation of MMP-2. Our animal model has progressive hyperparathyroidism and hyperphosphatemia and therefore we determined the direct effect of these on MMP-2 and MMP9 expression in VSMC from CKD and normal animals as both are known risk factors for cardiovascular disease and mortality [1]. In vitro, there was already increased expression of both MMPs in CKD compared to normal animals, but phosphorus only increased MMP expression in VSMC from normal animals. This could be interpreted to indicate that phosphorus does directly upregulate MMP-2 and MMP-9, but cannot further be upregulated in the setting of CKD. However, we did not observe changes in zymography so we cannot conclude that phosphorus activates MMPs, but rather that it may increase expression. Chung et al. [18] also found a positive correlation between patient serum phosphorus levels and MMP2 expression in the inferior epigastric arteries. Similarly, in CKD patients there was a positive relationship of hyperphosphatemia with serum MMP-2 levels [20]. However, we found increased tissue expression early in the course of CKD when the animals were not yet hyperphosphatemic. Multiple other factors are also known to increase MMP-2 expression from VSMC including C-reactive protein, elevated homocysteine, and inflammation [4]. MMP-9 can be induced by tumor necrosis factor, oxidized low-density lipoprotein, and extracellular matrix degradation products [4]. Thus, the inflammatory and oxidative stress of CKD may contribute to the increased MMP expression early in the course of CKD, with additive effects of hyperphosphatemia and/or positive phosphate load. A final possibility is that the genetic abnormality of the cilia in this animal model of cystic kidney disease may also lead to abnormal vascular remodeling that persisted in cell culture.

In conclusion, we have demonstrated a progressive increase in serum MMP-2 levels and activity and arterial expression of MMP-2 and MMP-9 with progressive CKD. This would lead to altered extracellular matrix and, consequently, altered arterial structure and function. The altered matrix likely forms a preferential nidus for the initiation of arterial calcification. Supporting this direct effect are studies demonstrating that inhibition of calcification occurs in ex vivo ring cultures with inhibitors of MMP and other animal data in non-CKD models. However, in vivo studies in CKD animal models and patients with CKD are needed to determine if preventing this extracellular matrix degradation also prevents arterial calcification in CKD.

\section{Acknowledgment}

This work was supported by a VA Merit Award to Dr. Moe.

\section{Disclosure Statement}

The authors have no conflicts of interest to disclose.
Chen/O’Neill/Chen/Kiattisunthorn/ Gattone/Moe 


\section{References}

1 Moe SM, Chen NX: Mechanisms of vascular calcification in chronic kidney disease. J Am Soc Nephrol 2008;19:213-216.

-2 Ortega N, Behonick DJ, Werb Z: Matrix remodeling during endochondral ossification. Trends Cell Biol 2004;14:86-93.

$\checkmark 3$ Lijnen HR: Metalloproteinases in development and progression of vascular disease. Pathophysiol Haemost Thromb 2003;33: 275-281.

4 Back M, Ketelhuth DF, Agewall S: Matrix metalloproteinases in atherothrombosis. Prog Cardiovasc Dis 2010;52:410-428.

$\checkmark 5$ Beaudeux JL, Giral P, Bruckert E, Foglietti MJ, Chapman MJ: Matrix metalloproteinases, inflammation and atherosclerosis: therapeutic perspectives. Clin Chem Lab Med 2004;42:121-131.

6 Pawlak K, Pawlak D, Mysliwiec M: Serum matrix metalloproteinase-2 and increased oxidative stress are associated with carotid atherosclerosis in hemodialyzed patients. Atherosclerosis 2007;190:199-204.

-7 Fondard O, Detaint D, Iung B, et al: Extracellular matrix remodelling in human aortic valve disease: the role of matrix metalloproteinases and their tissue inhibitors. Eur Heart J 2005;26:1333-1341. $\checkmark 8$ Liu PY, Tsai WC, Lin CC, Hsu CH, Haung $\mathrm{YY}$, Chen JH: Invasive measurements of pulse wave velocity correlate with the degree of aortic valve calcification and severity associated with matrix metalloproteinases in elderly patients with aortic valve stenosis. Clin Sci (Lond) 2004;107:415-422.

-9 Qin X, Corriere MA, Matrisian LM, Guzman RJ: Matrix metalloproteinase inhibition attenuates aortic calcification. Arterioscler Thromb Vasc Biol 2006;26:1510-1516.

10 Moe SM, Seifert MF, Chen NX, et al: R-568 reduces ectopic calcification in a rat model of chronic kidney disease-mineral bone disorder (CKD-MBD). Nephrol Dial Transplant 2009;24:2371-2377.

11 Moe SM, Chen NX, Seifert MF, et al: A rat model of chronic kidney disease-mineral bone disorder. Kidney Int 2009;75:176184.

12 El Bedoui J, Oak MH, Anglard P, SchiniKerth VB: Catechins prevent vascular smooth muscle cell invasion by inhibiting MT1-MMP activity and MMP-2 expression. Cardiovasc Res 2005;67:317-325.

13 Moe SM, Duan D, Doehle BP, O’Neill KD, Chen NX: Uremia induces the osteoblast differentiation factor Cbfal in human blood vessels. Kidney Int 2003;63:1003-1011.

14 Lomashvili KA, Cobbs S, Hennigar RA, Hardcastle KI, O’Neill WC: Phosphate-induced vascular calcification: role of pyrophosphate and osteopontin. J Am Soc Nephrol 2004;15:1392-1401.
15 Chen NX, Chen X, O’Neill KD, Atkinson SJ, Moe SM: RhoA/Rho kinase (ROCK) alters fetuin-A uptake and regulates the calcification in bovine vascular smooth muscle cells (BVSMC). Am J Physiol Renal Physiol 2010; 299:F674-F680.

$\checkmark 16$ Kieffer P, Giummelly P, Schjoth B, et al: Activation of metalloproteinase-2, loss of matrix scleroprotein content and coronary artery calcification. Atherosclerosis 2001;157: 251-254.

17 Basalyga DM, Simionescu DT, Xiong W, Baxter BT, Starcher BC, Vyavahare NR: Elastin degradation and calcification in an abdominal aorta injury model: role of matrix metalloproteinases. Circulation 2004;110: 3480-3487.

18 Chung AW, Yang HH, Kim JM, et al: Upregulation of matrix metalloproteinase- 2 in the arterial vasculature contributes to stiffening and vasomotor dysfunction in patients with chronic kidney disease. Circulation 2009; 120:792-801.

19 Chung AW, Yang HH, Sigrist MK, et al: Matrix metalloproteinase-2 and -9 exacerbate arterial stiffening and angiogenesis in diabetes and chronic kidney disease. Cardiovasc Res 2009;84:494-504.

-20 Peiskerova M, Kalousova M, Kratochvilova $\mathrm{M}$, et al: Fibroblast growth factor 23 and matrix metalloproteinases in patients with chronic kidney disease: are they associated with cardiovascular disease? Kidney Blood Press Res 2009;32:276-283. 